\title{
Next Generation Sequencing for Diagnostics of Myeloid Malignancies for Routine Clinical Use: Pitfalls and Advantages
}

The use of Next Generation Sequencing is being increasingly sought to be included in routine clinical use for more differentiated disease classification, risk stratification and therapeutic decisions, particularly in AML, MDS, and MPNs. But unexpected challenges are being faced towards interpretation and implementation of the results obtained.

Commercially available panels are based on targeted sequencing and usually analyze $25-50$ genes and generally add to the information routinely available on karyotyping, FISH, and Real Time PCR panels. In AML, more than one recurrent somatic mutation can be identified, in MDS in nearly $30 \%$ of cases (e.g., MDS with ring sideroblasts with SF3B1 mutation). In MPNs, however the significance of non-driver mutations, outside of driver mutations of JAK2, CALR and MPL are difficult to define. In triple negative MPNs, NGS may confirm the presence of a clonal disease. In spite of this, a large number of triple negative MPN patients show no detectable mutation. Another pitfall can be the discrimination of leukemia-initiating mutations from incidental passenger mutation or CHIP or germline mutations and requires extensive validation of the results in a large number of cases. Thus, the review of the reported genetic variants by experts with technical and clinical knowledge is highly relevant [1].

Shown here in the AML group are a representative number of four cases of AML, each different from the other with respect to cytogenetics and RT-PCR molecular findings. They were then analyzed by NGS. In these cases, it appears, that cytogenetics and RT-PCR investigation were adequate towards risk stratification and clinical decision making. While this may be true in many cases, it by no means diminishes the value of doing NGS to look for other mutations specially in AML and MDS, where nearly $50 \%$ of the patients are cytogenetically and by RT-PCR negative. This would be particularly true in the larger clinical context towards the use of targeted therapy.

\section{Reference}

1. Bacher U, Shumiov E, Flach J, et al. Challenges in the introduction of next generation sequencing (NGS) for diagnostic of myeloid malignancies into clinical routine use. Blood Cancer J. 2018;8:113. https://doi. org/10.1038/s41408-018-0148-6. 